\title{
THE EFFECTS OF OXYTETRACYCLINE CHRONIC TOXICITY ON THE POPULATION DYNAMICS OF D. MAGNA IN THE PRESENCE OF UV-B
}

\author{
LinARES GONZÁLEZ, Y. ${ }^{1}$ - LiNARES Fleites, G. ${ }^{2}$ - GARCÍA VARGAS, S. ${ }^{3}-$ \\ MARTÍNEZ CONTRERAS, R. ${ }^{4}-$ PEÑA MORENO, R. ${ }^{3}-$ MORALES, L. L. ${ }^{*}$ \\ ${ }^{1}$ Posgrado en Ciencias Ambientales, Instituto de Ciencias, Benemérita Universidad Autónoma \\ de Puebla, Edificio IC 6, Ciudad Universitaria, C. P. 72570 Puebla, México \\ ${ }^{2}$ Departamento de Investigación en Ciencias Agrícolas, Instituto de Ciencias, Benemérita \\ Universidad Autónoma de Puebla, Av. 4 Sur 104, C. P. 72000 Puebla, México \\ ${ }^{3}$ Centro de Química, Instituto de Ciencias, Benemérita Universidad Autónoma de Puebla, Av. 4 \\ Sur 104, C. P. 72000 Puebla, México \\ ${ }^{4}$ Laboratorio de Ecología Molecular Microbiana, Centro de Investigaciones en Ciencias \\ Microbiológicas, Instituto de Ciencias, Benemérita Universidad Autónoma de Puebla, Av. 4 Sur \\ 104, C. P. 72000 Puebla, México \\ ${ }^{5}$ Facultad de Ciencias Químicas, Benemérita Universidad Autónoma de Puebla, Av. 4 Sur 104, \\ C. P. 72000 Puebla, México \\ "Corresponding author \\ e-mail: laura.morales@correo.buap.mx \\ (Received 20 $0^{\text {th }}$ Sep 2019; accepted $8^{\text {th }}$ Jan 2020)
}

\begin{abstract}
Oxytetracycline is a broad-spectrum antibiotic which is mainly used to improve the health of aquaculture and livestock animals, which is the reason why it is released into water bodies through the discharges of these industries potentially causing an imbalance in the freshwater ecosystems. Additionally, the depletion of stratospheric ozone has caused an increase in the levels of ultraviolet radiation, which can increase the toxicity of the drug, due to the interaction between oxytetracycline and the UV-B region of the electromagnetic spectrum in particular, thus deteriorating the conditions necessary for the survival of aquatic species. This work analyzes the toxicity of oxytetracycline in presence of UV-B radiation using model organism Daphnia magna and the impact of these stressors on the population development, and expands the subject with a mathematical model that explores its effects on freshwater ecosystems. A chronic test was performed by combined exposures of oxytetracycline and UV-B radiation. Results showed that the effect of the stressors significantly decreased the growth rate coinciding with the alterations identified in the reproduction, thus modifying the population dynamics. This was simulated with mathematical modeling which shows that both oxytetracycline and UV-B radiation are factors that reduce survival and modify the reproductive cycle of the model organism.

Keywords: mathematical model, population structure, reproductive effects, carrying capacity, model organism
\end{abstract}

\section{Introduction}

Uncontrolled consumption of pharmaceutical products for human and veterinary uses has caused a raise in pollution levels within freshwater ecosystems (Heckmann et al., 2007) and represents an environmental risk for species that are sensible to biologically active substances. There has been much research focusing on antibiotics, given that their presence in water contributes to the propagation of microbial resistance 
(Segura et al., 2015), and that their interactions with other environmental factors causes a raise in toxicity. Such is the case of Tetracycline (TC), an antibiotic that is used regularly as a therapeutic agent and promoter of animal growth. The cyclic structure of these antibiotics allows them to absorb light from the ultraviolet region, thus making them photolabile (Chen et al., 2011; Liu et al., 2018).

Oxytetracycline (OTC) is an antibiotic in the TC group, it has been detected frequently in water bodies and sediments from several countries (Zhaoa et al., 2013), due to its low absorption level in organisms, especially in animals, its active form is released to the environment through urine and feces. It is estimated that approximately $1900 \mathrm{Kg}$ of OTC can reach surface water bodies yearly (Daghrir et al., 2013) with an average residence time of about 9 days in these ecosystems and without any alteration in its structure (Jeffrey et al., 2006; Leal et al., 2019). However, when OTC interacts with the ultraviolet region of solar light, especially with the UV-B portion (i.e. $320-$ $280 \mathrm{~nm}$ ), it fosters an increase in its toxicity, affecting aquatic organisms by lowering their productivity and reproduction, and it affects species such as phytoplankton, zooplankton, macroalgae, fish eggs, and larvae particularly (Häder et al., 2015).

Freshwater zooplankton is composed mainly by three taxonomic groups: Rotifer, Cladocera and Copepod. Daphnia magna (D. magna) is an herbivorous cladoceran, that is largely used as a model organism to assess the impact of environmental changes due to its key position within the trophic chain as a link between primary producers (phytoplankton) and higher-level consumers (fish). D. magna serves as an indicator for water quality given that there is a close relation between environmental factors and these organisms sensitivity to changes in their surroundings, may they be either chemical or physical (Nevesa et al., 2015).

This research focused on exploring the OTC toxicity when interacting with UV-B radiation through a chronic test on the model organism $D$. magna and the impact these factors would have in the population development of the aforementioned species. By using a mathematical model that considers the organisms life cycle, as well as survival and fertility rates, it was possible to predict the changes that can happen to the structure and functioning of the population within freshwater ecosystems (Nevesa et al., 2015).

\section{Materials and methods}

\section{Test organisms}

The organism employed for this study was D. magna, obtained from the Laboratory of Ecology and Restoration of Aquatic Systems in the Autonomous University of Puebla, in Puebla, Pue., Mexico. Daphnid cultures were started with 10 female neonates out of a batch of reproducers of known age. Groups composed by 20 daphnids were mantained in $1 \mathrm{~L}$ of reconstituted hard water (i.e. total hardness of $250 \mathrm{mg} / \mathrm{L}$ of $\mathrm{CaCO}_{3}$ ) with $\mathrm{pH}$ between $7-9$. The water was renewed twice per week. For the purposes of this study, 9 batches were implemented for asexual reproduction, and the offspring was in subsequent tests. The cultures were kept at room temperature with a 16:8 photoperiod (light:darkness) and fed every third day with a mix of Spirulina and yeast (TetraVeggieTM) in a 25,000 cells/mL concentration (Kim et al., 2009; Hall et al., 2012). 


\section{Experimental design}

\section{OTC toxicity test}

OTC is commercially available in several formulations that can be presented as pre-mixed food, injectables, soluble powder and tablets for animal use. The OTC used in this study was obtained as an injectable solution $\left(\mathrm{C}_{22} \mathrm{H}_{24} \mathrm{~N}_{2} \mathrm{O}_{9}\right)$ from NorVet (SAGARPA Q-7827-047, batch: 174140). In principle, OTC is soluble in water $(100 \mathrm{~g} / \mathrm{L})$ at $20^{\circ} \mathrm{C}$ either in acid or basic solutions (Baguer et al., 2000), it can also behave as a strong chelating agent by forming complexes with metallic ions such as $\mathrm{Ca}^{2+}$ and $\mathrm{Mg}^{2+}(\mathrm{Jeffrey}$ et al., 2006).

The ideal OTC concentration for this work was selected from acute and chronic toxicity tests. At first, the acute test method established in the literature (Gallina et al., 2008) was used to determine the OTC toxicity in daphnids cultured in the laboratory. The estimated $\mathrm{EC}_{50}$ for OTC at 48 hours was $0.2 \mathrm{mg} / \mathrm{mL}$ (Gallina et al., 2008). Subsequently, for a period of 28 days the populations were exposed to concentrations lower than the $\mathrm{EC}_{50}$ (i.e. $0.025 \mathrm{mg} / \mathrm{mL}, 0.010 \mathrm{mg} / \mathrm{mL}$ and $0.004 \mathrm{mg} / \mathrm{mL}$ ) with manual counting and medium replacement every third day; this was done to find a suitable subletal concentration for monitoring individuals in subsequent experiments. This pilot study showed impacts on the daphnid reproduction, especially for the group subjected to the $0.025 \mathrm{mg} / \mathrm{mL}$ concentration, which is why it was selected to carry out further tests.

\section{$U V$-B irradiation toxicity test}

A pilot test was carried out to select the UV-B radiation intensity, period of irradiation and the age at which daphnids should be irradiated. Survival tests were performed with a UV-B lamp (model UVB-313, INSTRULAMP). The containers enclosed 10 individuals of different ages (i.e. 48 hours, 4 days and 7 days) in $120 \mathrm{~mL}$ of reconstituted hard water in triplicate. They were placed at two different heights under the UV-B lamp (i.e. 10 and $20 \mathrm{~cm}$ ). The exposures were recorded several times, which included irradiation times of 10 and 20 minutes followed by monitoring after 24, 48, 72 and 96 hours had passed until the immobilization of less than $50 \%$ of the organisms was observed, and to allow subsequent follow-up. The UV intensity was measured during the exposure periods, using a specific radio device that was placed under the lamp to obtain information on the intensity provided in $\mathrm{W} / \mathrm{m}^{2}$.

On the other hand, to achieve a biologically relevant dose of UV radiation, a weighting factor was used according to the reference action spectrum of the International Commission on Illumination (ICI) for erythema on human skin (Eq. 1).

$$
E_{\text {erythema }}\left(J / m^{2}\right)=I\left(W / m^{2}\right) \times \varepsilon \times t(s)
$$

where:

$E_{\text {erythema }}$ stands for erythematic irradiation; $I$ stands for the spectral radiation from the source (UV lamp, model UVB-313 INSTRULAMP) in $\mathrm{W} / \mathrm{m}^{2} ; \varepsilon$ stands for the erythematic coefficient; and $t$ stands for time in seconds (Azevedo et al., 2016).

By using this equation, an effective dose of $75.57 \mathrm{~J} / \mathrm{m}^{2}$ was determined. In this way, when the spectrum of erythematic action of the ICI is used, the effective dose can also be expressed as the erythemal standard dose (ESD) with a numerical value of $100 \mathrm{~J} / \mathrm{m}^{2}$. For this work, an ESD of 0.75 was used. 


\section{OTC chronic test and $U V-B$ radiation}

The chronic test considered four groups in triplicate, each group contained twenty neonatal individuals within $120 \mathrm{~mL}$ of medium and were exposed to different conditions: one group was subjected to an OTC constant concentration of $0.025 \mathrm{mg} / \mathrm{mL}$; another one to UV-B radiation $\left(2.2 \mathrm{~W} / \mathrm{m}^{2}\right)$. The tests were delayed until the daphnids reached an age of seven days to ensure a larger survival rate. The containers were placed at 20 centimeters from the lamp (UVB-313 INSTRULAMP) for 10 minutes, they were then moved under different lamps (WCAM 207229) to provide them with a normal photoperiod. The same conditions were adopted for the group that considered the interaction between OTC and UV-B radiation. Lastly, there was a control group that was kept under standard conditions. These populations were observed over 20 days and monitored every third day by manual counting.

\section{Statistical methods}

All treatments were performed in triplicate and the results were reported as mean \pm standard deviation. Statistical analyzes were performed by generalized linear model adjustment with Poisson distribution using logarithms as a link function, then the Tukey test was carried out as a post-hoc test to determine the effects on the population parameters evaluated.

\section{Mathematical model}

To describe the population dynamics of D. magna, matrix population models were used. They project the population growth from time $t$ to time $t+1$ in terms of the vital rates during each life stage, focusing especially on the reproduction and survival (Webb et al., 2011), in this way a projection matrix (Eq. 2) is constructed:

$$
A n_{t}=n_{t+1}
$$

where:

$n$ is a vector $q x 1$ that describes the distribution of the population for each the life stage at time $t ; q$ is the number of stages; and $A$ is the population projection matrix, constructed from the decomposition of the life cycle of D. magna in categories (Fig. 1) to achieve a transition structure (Eq. 3) (Takada et al., 1992).

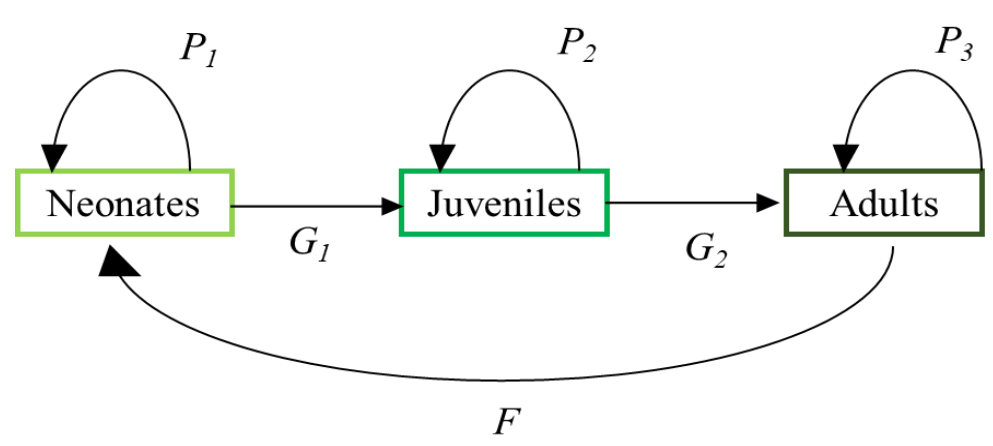

Figure 1. Life cycle of D. magna in which the population was classified in three stages: neonates, juveniles and adults. $P_{i}$ is the probability of surviving and staying in the same stage;

$G_{i}$ is the probability of surviving and growing onto the next stage; and $F$ is the fertility, calculated by observation period 


$$
A=\begin{array}{ccc}
P_{1} & 0 & F \\
G_{1} & P_{2} & 0 \\
0 & G_{2} & P_{3}
\end{array}
$$

where, $P$ is the probability of surviving and staying in Stage $i, G$, is the probability of surviving and growing into the next stage, and $F$, is the fertility per female per period. In addition, each of the elements of the equation were calculated from the population monitoring with the consequent elaboration of the life tables and considering the vital rates value (Eqs. 4 and 5).

$$
\begin{gathered}
G_{i j}=s_{j} g_{i j} \\
P_{i j}=s_{j}\left(1-g_{i j}\right)
\end{gathered}
$$

where:

$s_{j}$ : survival rate

$f_{j}$ : fertility rate

$g_{i j}$ : "growth" rate (phase transition).

Additionally, the observation of the populations supports the definition of fertility as a parameter that depends on the carrying capacity of the system $(\mathrm{K})$ as well as on the percentage of individuals of reproductive age.

The simulation and manipulation of the mathematical model was done with a computational program designed using the Visual Studio compiler (2017) in C++ language (Tokachi et al., 2019).

\section{Results}

\section{Chronic effect of OTC over reproduction}

The most significant effects in the OTC pilot study were observed in reproduction, given that the time to reach sexual maturity (i.e. the day of first reproduction) for the three exposed groups was delayed with respect to control group $(P<0.05)$. The group that was subjected to $0.025 \mathrm{mg} / \mathrm{mL}$ was the one with the most notable difference $(P=0.016)$. Moreover, a decrease in the number of released neonates per female was identified. These results are shown in Table 1.

Table 1. Reproduction and survival of D. magna after being exposed to OTC for 28 days

\begin{tabular}{c|c|c|c|c}
\hline $\begin{array}{c}\text { Assessed parameter } \\
\text { Time to reach sexual } \\
\text { maturity (d) }\end{array}$ & $8.60 \pm 0.57^{\mathrm{a}}$ & $10.60 \pm 0.57^{\mathrm{b}}$ & $9.60 \pm 0.50^{\mathrm{ab}}$ & $12.60 \pm .57^{\mathrm{c}}$ \\
\hline $\begin{array}{c}\text { Number of females } \\
\text { (Adults) }\end{array}$ & $73 \pm 2.83^{\mathrm{a}}$ & $72 \pm 8.80^{\mathrm{ac}}$ & $65 \pm 5.00^{\mathrm{d}}$ & $83 \pm 1.52^{\mathrm{c}}$ \\
\hline Number of neonates & $286 \pm 4.24^{\mathrm{a}}$ & $125 \pm 2.10^{\mathrm{c}}$ & $225 \pm 1.41^{\mathrm{b}}$ & $116 \pm 2.12^{\mathrm{c}}$ \\
\hline Number of juveniles & $142 \pm 0.73^{\mathrm{a}}$ & $115 \pm 141^{\mathrm{b}}$ & $132 \pm 2.12^{\mathrm{c}}$ & $96 \pm 2.83^{\mathrm{a}}$ \\
\hline Number of dead & $14 \pm 1.41^{\mathrm{a}}$ & $12.50 \pm 0.70^{\mathrm{a}}$ & $13 \pm 1.41^{\mathrm{a}}$ & $16.50 \pm 0.73^{\mathrm{b}}$ \\
\hline
\end{tabular}

The table reports mean values \pm standard deviation. The letters indicate significant differences $(\mathrm{P}<0.05)$ 


\section{$U V-B$ radiation preliminary test}

The results for the pilot testing (Fig. 2) correspond to the variation between distance and exposure times to which the individuals of different ages were subjected, while keeping light intensity constant $\left(2.2 \mathrm{~W} / \mathrm{m}^{2}\right)$. Higher mortality rates were recorded in the younger groups, neonates and juveniles $(P<0.05)$, thus demonstrating the vulnerability of these organisms to the minimum exposure time.

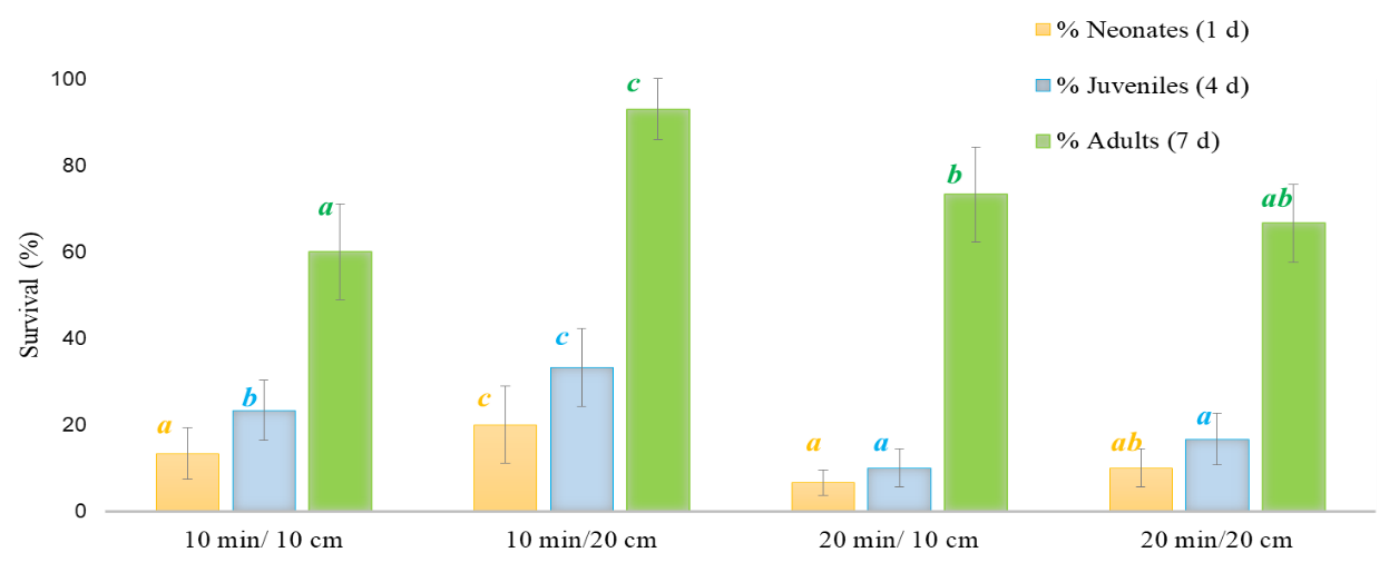

Figure 2. Toxicity test for D. magna subjected to UV-B radiation for different time periods and distances. Each bar represents the survival percentage for each age group, depending on the conditions to which they were subjected. The letters indicate significant differences $(P<0.05)$

Similarly, the statistical analysis allowed to rule out the influence of the type of treatment on survival. However, given the obtained data, the highest survival percentage for the three age groups was registered for individuals exposed at $20 \mathrm{~cm}$ separation from the lamp for 10 minutes. Thus, these conditions were chosen, despite not having registered significant differences $(P=0.06)$.

\section{Population dynamic alterations due to OTC and UV-B}

One of the objectives of this study was to identify the toxicity of OTC in interaction with UV-B radiation by means of a chronic test that evidences the impact on the population development of $D$. magna by using survival and fertility rates as parameters that describe the population behavior. In the case of reproduction (Table 2) one of the most significant effects was the delay of the first laying $(P<0.05)$ for the three treatments compared to the control group; however, this delay was more evident in the groups subjected to OTC $(P=0.0012)$ and both stressors $(P=0.0037)$. This result, however, does not depend on the interaction between the antibiotic and UV-B irradiation; for the treatment that included both, OTC and UV-B, the first reproduction happened before (i.e. day 12) than in the treatment that had OTC only (i.e. day 13).

Total reproduction (i.e. total number of births over 21 days) was assessed, resulting in a negative relationship depending on the type of treatment, showing a decrease in the number of neonates for each group according to the type of stressor (Table 2). Similarly, when compared to the control group all the treatments present significant differences $(P<0.05)$. However, the largest impact is seen in the group subjected to constant concentration of OTC $(0.025 \mathrm{mg} / \mathrm{mL})$ where the total number of neonates is $70 \%$ lower 
compared to the control group, coinciding with the decrease in fertility observed in the trials. All in all, the average number of neonates that a female had throughout her life was significantly reduced in all treatments, when compared to the control group $(P<0.05)$.

Table 2. Reproduction and survival rates for D. magna after 21 days

\begin{tabular}{c|c|c|c|c}
\hline \multirow{2}{*}{ Assessed parameter } & \multicolumn{4}{|c}{ Conditions of the experiment } \\
\cline { 2 - 5 } & Control & $\begin{array}{c}\mathbf{0 . 0 2 5} \mathbf{~ m g} \\
\text { OTC/mL }\end{array}$ & UV-B & $\begin{array}{c}\text { 0.025 mg OTC/mL } \\
\text { and UV-B }\end{array}$ \\
\hline $\begin{array}{c}\text { Time to reach sexual } \\
\text { maturity (d) }\end{array}$ & $8.6 \pm 0.57^{\mathrm{a}}$ & $13 \pm 0^{\mathrm{b}}$ & $11.60 \pm 1.15^{\mathrm{b}}$ & $12.30 \pm 1.15^{\mathrm{b}}$ \\
\hline $\begin{array}{c}\text { Total reproduction (Total } \\
\text { number of neonates) }\end{array}$ & $380.60 \pm 46.90^{\mathrm{a}}$ & $105 \pm 19.92^{\mathrm{b}}$ & $285.31 \pm 10.01^{\mathrm{c}}$ & $179.60 \pm 18.14^{\mathrm{d}}$ \\
\hline Neonates per female & $5.60 \pm 0.57^{\mathrm{a}}$ & $3.14 \pm 0.16^{\mathrm{cb}}$ & $4 \pm 0.14^{\mathrm{ba}}$ & $2.80 \pm 0.25^{\mathrm{c}}$ \\
\hline Number of molts & $266.30 \pm 4.72^{\mathrm{a}}$ & $169 \pm 9.84^{\mathrm{c}}$ & $193.60 \pm 9.45^{\mathrm{b}}$ & $181 \pm 10.14^{\mathrm{cb}}$ \\
\hline
\end{tabular}

The table reports mean values \pm standard deviation. The letters indicate significant differences $(\mathrm{P}<0.05)$

On the other hand, individuals were classified in three stages of development (i.e. neonates, juveniles and adults) to assess alterations in the population structure induced by the physicochemical factors that were being studied (Fig. 3). The results show the impact of both stressors on the populations by registering high percentages of adults for all the treatments compared to the control group. In the OTC and UV-B group the relative percentage of adults doubled, a fact that in conjunction with the decrease in fertility, can risk the survival of the population over time.

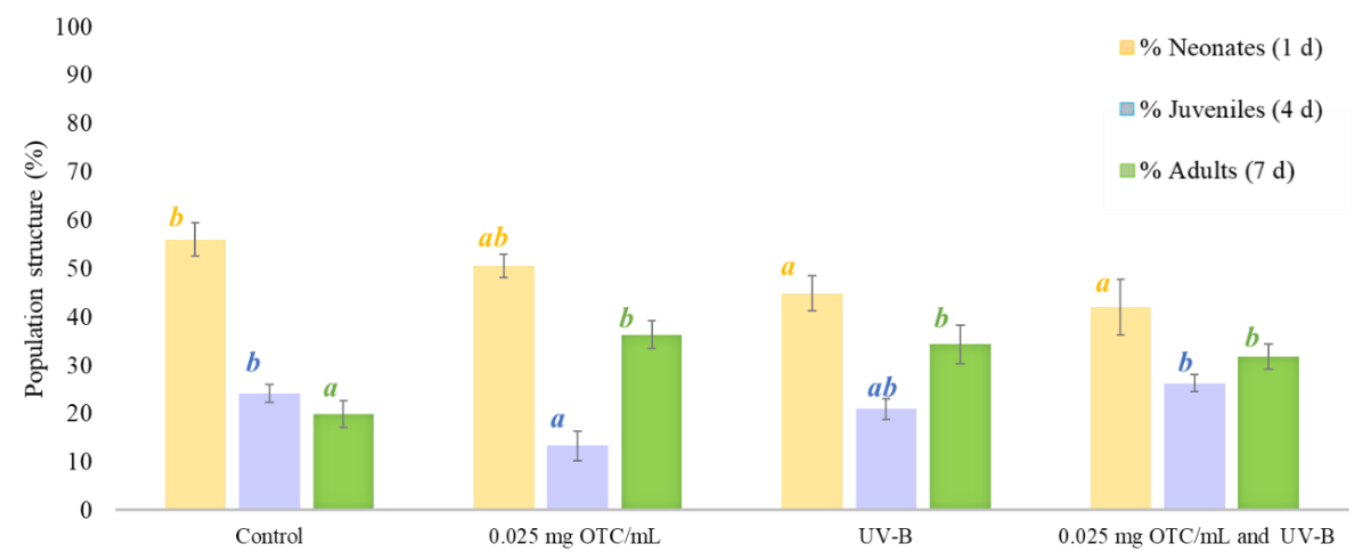

Figure 3. Distribution of D. magna populations in three age groups after 20 days of treatment (mean value \pm standard deviation). Different letters indicate significant differences $(P<0.05)$

\section{Projection of the population dynamics through mathematic modeling}

The population projection for D. magna in a simulated control group ( $\mathrm{K}=600$ individuals) indicates an average monthly growth of $13 \%$ and a mortality of $3.5 \%$ (Floydand et al., 1988; Lo et al., 1995; Coll et al., 2019) with a net reproductive rate $\left(\mathrm{R}_{0}\right)$ of 6.2, and a generation time (T) of 3 days (Meyer et al., 1986). The age distribution, 
which corresponds to the population structure, is similar to the one observed in the control groups, with a population composed mostly by neonates and juveniles, maintaining a stable reproduction rate and ensuring the survival of the population over time (Fig. 4).

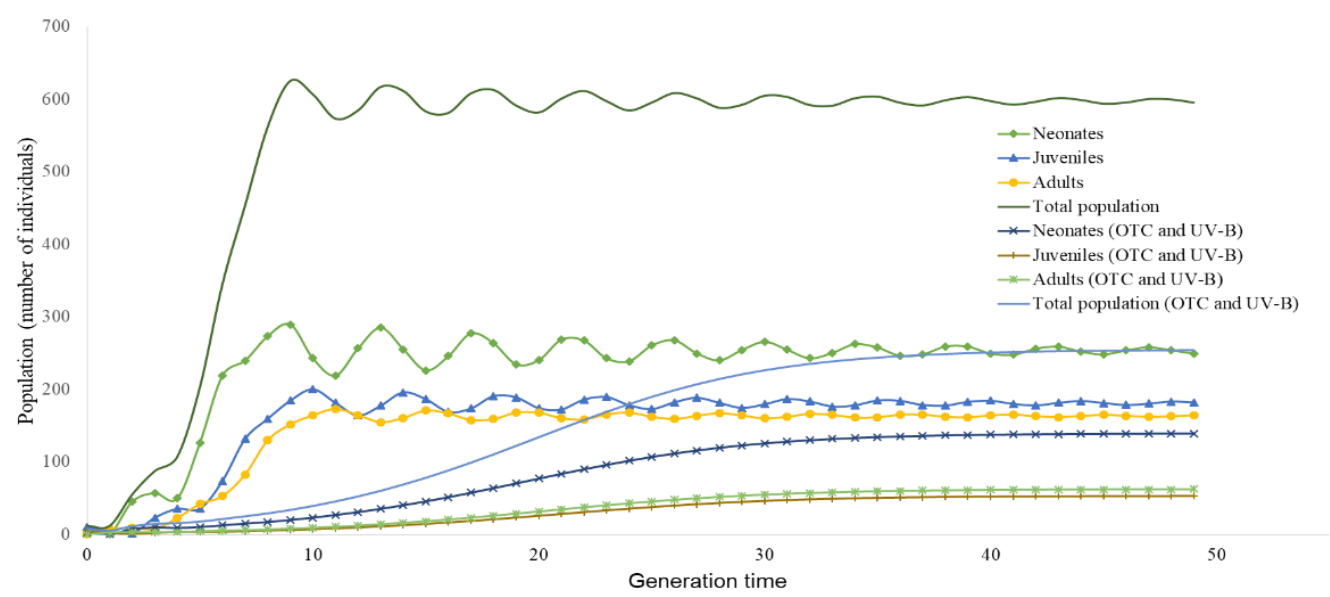

Figure 4. Simulation of the population dynamics of $D$. magna for the control group and the group subjected to OTC and $U V-B$

Conversely, the population projection for organisms subjected to chronic exposure of oxytetracycline in the presence of UV-B, while keeping the population size $(K=600$ individuals), presents a monthly average growth of $7.3 \%$ and a mortality of $4.5 \%$ (Floydand et al., 1988; Lo et al., 1995; Coll et al., 2019). The value of $\mathrm{R}_{0}$ was 2.8 with $\mathrm{T}$ of 3 days, therefore, the average number of neonates per gravid female that integrate into the population every third day was diminished by $54 \%$ with respect to the control group, thus altering the population structure because of the relative increase in the number of adults, in comparison with the quantities of juveniles and neonates due to a decline in reproduction rates (Fig. 4) (Meyer et al., 1986; Bernhardt et al., 2018). The registered changes on the finite population growth rate show negative values, which can account for the decline in a population that might as well be facing extinction (Heckmann et al., 2007).

\section{Discussion}

The results of this study show the consequences over D. magna populations when exposed to OTC concentrations of environmental relevance for a certain time period, specific effects were observed on parameters associated with reproduction: the population structure was altered as a consequence of delays in sexual maturity and the reduction in the number of neonates per female, which also decreased the number of juveniles.

This reproductive effect is supported by the "Principle of allocation" according to which the energy obtained by an individual is divided among the different requirements for maintenance, growth and reproduction. Any extra energy spent in any one of the aforementioned requirements will result in less available energy for the other two. Therefore, the decrease in reproduction would allow for some energy to be invested in the maintenance and survival of organisms (Mollet et al., 2002; Heckmann et al., 2007). This effect has been reported in various ecotoxicological studies, such as Koivisto et al. 
(1995) and Eluk et al. (2017), which agree on the importance of assessing not only mortality as an alert parameter but also the reproductive effects, like important bioindicators in order to infer possible damage to aquatic communities in water bodies receiving residual discharges (Rautiainen et al., 2004).

It should be noted that no synergism was observed among the assessed stressors, because the effect of UV-B radiation is covered by the presence of OTC. Some studies have revealed that $\mathrm{TC}$ can cause a higher production of melanin and other complexes (Rok et al., 2015), a phenomenon that might explain the fact that daphnids treated with UV-B and OTC are, apparently, less damaged (Table 2) than the groups that were treated with any of the stressors individually; given that melanin is a compound that grants protection against adverse environmental conditions, such as UV radiation. However, it is important to highlight the chronic changes in the population structure (Fig. 3) caused by the presence of UV-B and OTC, which cause late reproduction and the reduction in the number of neonates per female, which in turn also decreases the number of juveniles and causes a decline in the population due to the inability to reproduce (Heckmann et al., 2007).

In contrast, the observations of the different treatments admit the incorporation of $\mathrm{K}$ as a parameter that, along with the percentage of individuals of reproductive age, define fertility and therefore the number of neonates that are integrated into the population. By considering these factors together, the simulations of population dynamics of D. magna become more realistic than models that are solely based on the population structure and their vital rates as demonstrated by Lo et al. (1995). It is also worth to mention that the incorporation of changes in somatic duration within the matrix model achieves more realistic projections, when comparing them to models that suppose a fixed duration for the various growth stages.

These results show the importance of adding convenient variables to the matrix models in order to get better explanations to evaluate chronic toxicity of the studied phenomenon. In this study the simulations were compared with monitored groups, which showed an exponential growth that tended to stabilize; however, for the group of interacting OTC and UV-B said stabilization occurred abruptly and without reaching the $\mathrm{K}$ value, mainly because of failures in reproductive events (Metcalf et al., 2015) that resulted in an $\mathrm{R}_{0}=2.8$, with a $54 \%$ decrease in the total number of neonates, when compared to the control group. Therefore, the population is far from an equilibrium since the birth rate cannot reach the mortality rate (4.5\%), thus destabilizing the population structure (Fig. 4).

\section{Conclusions}

This study has demonstrated that anthropogenic pollutants of the TC type, and OTC in particular, can have negative effects on the reproduction of D. magna, a species that is sensitive to physical and chemical alterations caused by their presence in fresh water. Some of these effects include delays in the sexual maturity of daphnids, as well as a reduction in birth rates, decreasing the number of neonates that integrate into the population and altering population dynamics. This scenario was verified through simulations supported by mathematical modeling, that showed that both OTC and UV-B radiation are limiting factors to the population growth and that they reduce the survival rates and modify the reproductive cycle of the organism.

In contrast, with the introduction of parameters that define the population dynamics into matrix models, it is possible to obtain detailed simulations of the population behavior 
of D. magna under realistic environmental conditions that represent a useful tool for the interpretation of later empirical studies. In future researches it will be necessary the development of chronic studies which allow the evaluation of population dynamics, through mathematical models which incorporate another variables of aquatic ecosystems such as $\mathrm{pH}$, hardness, temperature, dissolved oxygen, which could lead to increased toxicity induced by physicochemical factors OTC and UVB, in order to achieve a better understanding and evaluation of the environmental risk of these pollutants.

\section{REFERENCES}

[1] Azevedo, S. L., Ribeiro, F., Jurkschat, K., Soares, A. M., Loureiro, S. (2016): Co-exposure of $\mathrm{ZnO}$ nanoparticles and UV radiation to Daphnia magna and Danio rerio: Combined effects rather than protection. - Environmental Toxicology and Chemistry 35(2): 458-67.

[2] Baguer, A. J., Jensen, J., Henning, K. P. (2000): Effects of the antibiotics oxytetracycline and tylosin on soil fauna. - Chemosphere 40(7): 751-757.

[3] Bernhardt, J. R., Sunday, J. M., Thompson, P. L., O’Connor, M. I. (2018): Nonlinear averaging of thermal experience predicts population growth rates in a thermally variable environment. - The royal society publishing 285(1886): 1-10.

[4] Chen, Y., Li, H., Wang, Z., Tao, T., Hu, C. (2011): Photoproducts of tetracycline and oxytetracycline involving self-sensitized oxidation in aqueous solutions: Effects of $\mathrm{Ca}^{2+}$ and $\mathrm{Mg}^{2+}$. - International Journal of Environmental Science and Technology 23(10): 16341639.

[5] Coll, C., Sáncheza, E. (2019): Parameter identification and estimation for stage-structured population models. - International Journal of Applied Mathematics and Computer 29(2): 327-336.

[6] Daghrir, R., Drogui, P. (2013): Tetracycline antibiotics in the environment: a review. Environmental Chemistry Letters 11(13): 209-227.

[7] Eluk, D., Althaus, R., Nagel, O., Reno, U., Gagneten, A. M. (2017): Uso de Daphnia magna -organismo no target- para evaluar el impacto ambiental de quinolonas. - V Jornada de difusión de la investigación y extension.

[8] Floydand, S. K., Ranker, T. A. (1998): Analysis of a transition matrix model for Gaura neomexicana ssp. coloradensis (Onagraceae) reveals spatial and temporal demographic variability. - International Journal of Plant Sciences 159(5): 853-863.

[9] Gallina, G., Poltronieri, C., Merlanti, R., De Liguoro, M. (2008): Acute toxicity evaluation of four antibacterials with Daphnia magna. - Veterinary Research Communications 32: 287-290.

[10] Hall, M. D., Ebert, D. (2012): Disentangling the influence of parasite genotype, host genotype and maternal environment on different stages of bacterial infection in Daphnia magna. - Proc Biol Sci 279(1741): 3176-83.

[11] Häder, D. P., Kumar, H. D., Smith, R. C., Worrest, R. C. (2015): Effects of UV radiation on aquatic ecosystems and interactions with other environmental factors. - Photochemical \& Photobiological Sciences 14: 108-106.

[12] Heckmann, L. H., Callaghan, A., Hooper, H. L., Connon, R., Hutchinson, T. H., Maund, S. J., Sibly, R. M. (2007): Chronic toxicity of ibuprofen to Daphnia magna: Effects on life history traits and population dynamics. - Toxicology Letters 172(3): 137-145.

[13] Jeffrey, J., Werner, J. J., William, A. A., Mcneill, K. (2006): Water Hardness as a Photochemical Parameter: Tetracycline Photolysis as a Function of Calcium Concentration, Magnesium Concentration, and $\mathrm{pH}$. - Environmental Science \& Technology 40(23): 7236-7241. 
[14] Kim, J., Lee, M., Oh, S., Ku, J. L., Kim, K. H., Choi, K. (2009): Acclimation to ultraviolet irradiation affects UV-B sensitivity of Daphnia magna to several environmental toxicants. - Chemosphere 77(11): 1600-1608.

[15] Koivisto, S. (1995): Is Daphnia magna an ecologically representative zooplankton species in toxicity tests? - Environmental Pollution 90(2): 263-267.

[16] Leal, J. F., Esteves, V. I., Santos, E. B. H. (2019): Solar photodegradation of oxytetracycline in brackish aquaculture water: New insights about effects of $\mathrm{Ca} 2+\mathrm{and} \mathrm{Mg}$. - Journal of Photochemistry \& Photobiology A: Chemistry 372: 218-225.

[17] Liu, X., Lu, S., Guo, W., Xi. B., Wang, W. (2018): Antibiotics in the aquatic environments: A review of lakes, China. - Science of the Total Environment 627: 1195-1208.

[18] Lo, N., Smith, P., Butler, J. (1995): Population growth of northern anchovy and Pacific sardine using stage-specific matrix models. - Marine Ecology Progress Series 127(1): 1526.

[19] Metcalf, C. J. E., Ellner, S. P., Childs, D. Z., Gomez, R. S., Merow, C., McMahon, S. M., Jongejans, E., Rees, M. (2015): Statistical modelling of annual variation for inference on stochastic population dynamics using Integral Projection Models. - Methods in Ecology and Evolution 6: 1007-1017.

[20] Meyer, J. S., Ingersoll, C. G., McDonald, L. L., Boyce, M. S. (1986): Estimating Uncertainty in Population Growth Rates: Jackknife vs. Bootstrap Techniques. - Ecology 67(5): 1156-1166.

[21] Mollet, H. F., Cailliet, G. M. (2002): Comparative population demography of elasmobranchs using life history tables, Leslie matrices and stage-based matrix models. Marine and Freshwater Research 53: 503-516.

[22] Nevesa, M., Castroa, B. B., Vidala, T. T., Marques, J. C., Coutinho, J. A. P., Goncalves, F., Goncalves, A. M. M. (2015): Biochemical and populational responses of an aquatic bioindicator species, Daphnia longispina, to a commercial formulation of a herbicide (Primextra ${ }^{\circledR}$ Gold TZ) and its active ingredient (S-metolachlor). - Ecological Indicators 53: 220-230.

[23] Rautiainen, P., Laine, A. L., Aikio, S., Aspi, J., Siira, J., Hyvärinen, M. (2004): Seashore Disturbance and Management of the Clonal Arctophila fulva: Modelling Patch Dynamics. - Applied Vegetation Science 7(2): 221-228.

[24] Rok, J., Buszman, E., Delijewski, M., Otręba, M., Beberok, A., Wrześniok, D. (2015): Effect of tetracycline and UV radiation on melanization and antioxidant status of melanocytes. - Journal of Photochemistry and Photobiology B: Biology 148: 168-173.

[25] Segura, P. A., Takada, H., Correa, J. A., El Saadi, K., Koike, T., Agyeman, S. O., OfosuAnime, J., Sabi, E. B., Wasonga, O. V., Mghalu, J. M., Dos Santos Junior, A. M., Newman, B., Weerts, S., Yargeau, V. (2015): Global occurrence of anti-infectives in contaminated surface waters: Impact of income inequality between countries. - Environment International 80: 89-97.

[26] Takada, T., Nakajima, H. (1992): An analysis of life history evolution in terms of the density-dependent Lefkovitch matrix model. - Mathematical Biosciences 112(1): 155-176.

[27] Tokachil, M. N., Yahya, A. (2019): The Lefkovitch Matrix of Aedes Aegypti with Rainfall Dependent Model for Eggs Hatching. - Journal of Physics 1366: 1-8.

[28] Webb, A. R., Slaper, H., Koepke, P., Schmalwieser, A. W. (2011): Know your standard: clarifying the CIE erythema action spectrum. - Photochemistry and Photobiology 87(2): 483-486.

[29] Zhaoa, C., Pelaez, M., Duan, X., Denga, H., O’Shea, K., Fatta-Kassinos, D., Dionysiou, D. D. (2013): Role of $\mathrm{pH}$ on photolytic and photocatalytic degradation of antibiotic oxytetracycline in aqueous solution under visible/solar light: Kinetics and mechanism studies. - Applied Catalysis B: Environmental 134: 83-92. 